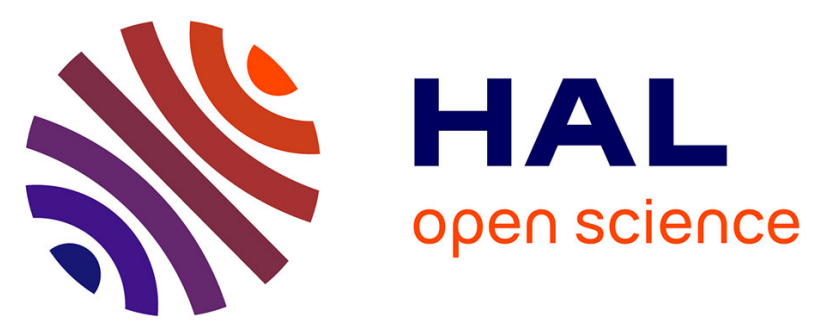

\title{
Electric field induced second harmonic (E-FISH) generation for characterization of fast ionization wave discharges at moderate and low pressures
}

Tat Loon Chng, Inna Orel, Svetlana Starikovskaia, I.V. Adamovich

\section{- To cite this version:}

Tat Loon Chng, Inna Orel, Svetlana Starikovskaia, I.V. Adamovich. Electric field induced second harmonic (E-FISH) generation for characterization of fast ionization wave discharges at moderate and low pressures. Plasma Sources Science and Technology, 2019, 28 (4), 045004 (8pp). 10.1088/13616595/ab0b22 . hal-02406807

\section{HAL Id: hal-02406807 https://hal.science/hal-02406807}

Submitted on 22 Nov 2020

HAL is a multi-disciplinary open access archive for the deposit and dissemination of scientific research documents, whether they are published or not. The documents may come from teaching and research institutions in France or abroad, or from public or private research centers.
L'archive ouverte pluridisciplinaire HAL, est destinée au dépôt et à la diffusion de documents scientifiques de niveau recherche, publiés ou non, émanant des établissements d'enseignement et de recherche français ou étrangers, des laboratoires publics ou privés. 


\title{
Electric Field Induced Second Harmonic (E-FISH) Generation for Characterization of Fast Ionization Wave Discharges at Moderate and Low Pressures
}

\author{
T.L. Chng ${ }^{1}$, I. S. Orel ${ }^{1}$, S. M. Starikovskaia ${ }^{1}$, and I.V. Adamovich ${ }^{2}$ \\ ${ }^{1}$ Laboratory of Plasma Physics (CNRS, Ecole Polytechnique, Sorbonne Universities, University of \\ Pierre and Marie Curie - Paris 6, University Paris-Sud), Ecole Polytechnique, route de Saclay, \\ 91128 Palaiseau, France \\ ${ }^{2}$ Nonequilibrium Thermodynamics Laboratories, Department of Mechanical and Aerospace \\ Engineering, Ohio State University, Columbus, OH 43210, USA
}

\begin{abstract}
The electric field in an ionization wave discharge in nitrogen at 20-100 mbar, initiated by positive polarity, high-voltage, ns duration pulses, is measured by ps second harmonic generation. The axial electric field component is determined both during the propagation of the ionization wave along the discharge tube, and after the wave reaches the grounded electrode, spanning the entire discharge gap. The temporal resolution of the present measurements is $200 \mathrm{ps,} \mathrm{with} \mathrm{the}$ spatial resolution in the axial direction of approximately $0.5 \mathrm{~mm}$. The second harmonic signal exhibits a quadratic dependence on the Laplacian electric field but indicates that in this pressure range most of the signal is generated within the wall of the tube. Absolute calibration of the signal is obtained from the current shunt data, after the ionization wave has reached the grounded electrode. Comparison of the data taken at different pressures shows that the peak value of the axial electric field in the wave front, $8-11 \mathrm{kV} / \mathrm{cm}$, has a fairly weak dependence on pressure, with the peak reduced electric field reaching $\approx 2000 \mathrm{Td}$ at 20 mbar. Reducing the pressure from 100 to 20 mbar, while keeping the discharge pulse voltage waveform the same, steepens the ionization wave front considerably, from $3.0 \mathrm{~ns}$ to $1.0 \mathrm{~ns}$ full width at half maximum. The results demonstrate that ps second harmonic generation may be employed for electric field measurements in lowpressure discharges, discharges sustained in small diameter capillary tubes, and discharges sustained in gas mixtures with low nonlinear susceptibility, at the conditions when the detection of the signal generated directly in the plasma is challenging. High temporal resolution of the present measurements indicates a possibility of detection of non-local electron kinetics effects induced by a rapidly varying, high peak value electric field in low and moderate pressure ionization wave discharges.
\end{abstract}




\section{Introduction}

Discharges generated by high peak voltage, ns duration pulses have been studied extensively over the last two decades, for applications including plasma-assisted combustion [13], plasma chemical fuel reforming [4] and $\mathrm{CO}_{2}$ dissociation [5], high-speed flow control [6], and plasma medicine [7]. Nanosecond pulse discharges generate nonequilibrium low-temperature plasmas over a wide range of pressures, from below a mbar [8,9] to several bar [10,11]. At moderate pressures, from several mbar to several tens of mbar, the discharge develops as a Fast Ionization Wave (FIW), kinetics of which are reviewed in great detail in [12,13]. A recent study of the FIW discharge in a capillary tube in air at 30 mbar [14] demonstrates that it produces nearly complete dissociation of molecular oxygen, suggesting that it can be used for the efficient plasma chemical generation of highly reactive radical species.

One of the most challenging aspects of characterization of kinetic processes in these plasmas, which occur on ns or even sub-ns time scales, is accurate diagnostics of electric field, electron density and temperature, and species number densities. A widely used method of electric field measurements in fast ionization wave discharges is by a calibrated capacitive probe $[15,16]$, with a temporal resolution up to $\sim 1 \mathrm{~ns}$ but a relatively low spatial resolution, $\sim 1 \mathrm{~cm}$. More recently, development and use of electro-optic probes has resulted in high temporal resolution $(\sim$ $1 \mathrm{~ns})$, high spatial resolution $(\sim 1 \mathrm{~mm})$ field measurements in atmospheric pressure plasma jets [17]. However, the presence of the probe perturbs the plasma to some extent, especially at the conditions when surface charge accumulation may occur on the probe housing. Development of non-intrusive, field vector sensitive, high spatio-temporal resolution diagnostics is essential for quantitative understanding of ionization kinetics in the wave, charge transport and species generation in the plasma behind the wave front, and wave propagation and attenuation.

The objective of the present work is to measure the electric field in a fast ionization wave, ns pulse discharge in nitrogen using the recently developed fs/ps Electric Field Induced Second Harmonic (E-FISH) diagnostic [18,19]. The principal advantage of this approach is sub-ns time resolution, limited primarily by the sampling rate of the detector and the oscilloscope. Previously, ps and fs E-FISH measurements have been done in atmospheric pressure ns pulse discharge plasmas in air, hydrogen, and argon [20-22], as well in a ns pulse discharge in 100 Torr of air [19]. In the latter set of measurements, the onset of the ionization wave propagating between the discharge electrodes has been detected. However, it appears that in these experiments the wave did not fully develop, most likely due to a relatively short discharge gap, $1.3 \mathrm{~cm}$. In the present work, a ns pulse discharge in nitrogen at 20-100 mbar is generated in a quartz tube between the electrodes set $8.0 \mathrm{~cm}$ apart, such that the development and propagation of the ionization wave could be studied in greater detail, over a wide pressure range.

\section{Experimental}

Figure 1 shows a schematic of the experimental apparatus. The discharge is sustained in a quartz tube with an internal diameter of $20 \mathrm{~mm}$, between two hollow truncated cone brass electrodes, as illustrated in Fig. 1. The gap between the electrodes is $8.0 \mathrm{~cm}$. Nitrogen is flown through the tube at the flow rate of $10 \mathrm{sccm}$ and the pressure of 20-100 mbar. The discharge in the tube is initiated using positive polarity high-voltage pulses, with peak voltage of $9.3 \mathrm{kV}$, pulse rise/fall time of $4 \mathrm{~ns}$, and pulse duration (FWHM) of $30 \mathrm{~ns}$, at a repetition rate of $10 \mathrm{~Hz}$, produced 
by an FPG 12-1NM (FID GmbH) high voltage pulse generator. The incident, reflected, and transmitted voltage pulse shapes are monitored by two calibrated, high bandwidth current shunts [24] respectively installed in the coaxial transmission line $12.5 \mathrm{~m}$ before the high-voltage electrode and $12.5 \mathrm{~m}$ after the grounded electrode (see Fig. 1). Each current shunt represents an approximately $2 \mathrm{~cm}$ long insertion into the coaxial cable shielding comprising of several low induction resistors soldered in parallel. The voltage drop across the current shunt is a timeaccurate, amplitude-scaled waveform of the actual high-voltage pulse moving along the line as discussed in detail in our previous work [25]. The distribution of the electric potential $\varphi(x, t)$ along the discharge gap is measured by a calibrated, high bandwidth, custom-made capacitive probe [15,23], a capacitive divider based on a low induction coaxial capacitor. The probe is moved along a slit machined in a cylindrical copper screen surrounding the discharge tube. The axial electric field $E_{x}(t)=\partial \varphi(x, t) / \partial x$ is calculated by measuring the electric potential at different points along the length of the tube with a step size of $1 \mathrm{~mm}$. Broadband plasma emission images are taken using a Princeton Instruments PI-MAX 4 ICCD camera, and the ionization wave speed along the discharge tube is inferred from the emission images by tracking the time evolution of the wave front development. The camera gate width for these experiments is set at $1.17 \mathrm{~ns}$, limited by the performance of the camera. Sequential emission images are captured every $0.2 \mathrm{~ns}$ for pressures 20, 40 and 70 mbar, and every $1 \mathrm{~ns}$ for the case of 100 mbar. The longer time delay chosen in the latter case is due to the slower ionization wave speed. The images are acquired from the moment of initiation of the ionization wave at the high-voltage electrode until its arrival at the grounded electrode, i.e. when the inter-electrode gap is closed. The position of the wave front, determined by locating the position of maximum emission intensity in each image, is plotted as a function of time, and the ionization wave speed is determined from the slope of this curve.

The schematic of the ps E-FISH diagnostic, also shown in Fig. 1, is similar to the one used in Ref. [19]. Briefly, the vertically polarized $1064 \mathrm{~nm}$ fundamental output beam of a ps laser (EKSPLA PL2241, pulse duration 30 ps, pulse energy $6 \mathrm{~mJ}$ ) is rotated to horizontal using a halfwave plate, and is directed through the quartz discharge tube halfway between the high-voltage and grounded electrodes, near its horizontal plane of symmetry, as shown schematically in Fig. 1. The beam is focused in the center of the tube by a $20 \mathrm{~cm}$ focal distance lens. The confocal parameter of the lens, assuming a Gaussian laser beam, is estimated to be $b \approx 1 \mathrm{~mm}$. No optical access windows are used to pass the laser beam through the discharge tube. The laser pulse energy is kept sufficiently low, around $6 \mathrm{~mJ}$, to prevent damage of the tube by the laser beam. A long pass filter, placed immediately after the first focusing lens, is used to block the stray second harmonic signal generated in the optical system components prior to the discharge tube. After exiting the discharge tube, the laser beam is collimated by a second $20 \mathrm{~cm}$ focal distance lens, and the second harmonic signal is separated from the fundamental beam using a dichroic mirror and a dispersive prism, as shown in Fig. 1. The intensity of the residual $1064 \mathrm{~nm}$ is monitored by a photodiode (Thorlabs DET10A, rise time $1 \mathrm{~ns}$ ). The $532 \mathrm{~nm}$ second harmonic beam passes through a polarizer to isolate the horizontally polarized signal proportional to the square of the axial electric field in the discharge, and is focused with a $10 \mathrm{~cm}$ focal length lens into the IR H7422-50P Hamamatsu photomultiplier module with a rise time of $1 \mathrm{~ns}$. An iris and $532 \mathrm{~nm}$ bandpass filter (FWHM 10 $\mathrm{nm})$ are placed at the entrance of the PMT for the purpose of stray light rejection. The photodiode and PMT signals are monitored by a digital oscilloscope (LeCroy WaveRunner 64Xi-A) with a 5 
$\mathrm{GHz}$ sampling rate and $600 \mathrm{MHz}$ bandwidth. For the lowest pressure of $20 \mathrm{mbar}$, the time-resolved plasma emission detected by the PMT during the discharge pulse (emission background) is measured with the laser turned off, and subtracted from the PMT signal measured with the laser on (see Section 3).

In spite of attempting to achieve sub-ns synchronization, there was an approximately 30 ns timing jitter between the laser and the high-voltage pulses. In order to address this issue, at each operating condition, a data acquisition strategy was employed whereby photodiode and PMT waveforms are saved for every laser shot (typically 40,000 shots per run). This procedure results in an ensemble of laser shots which are randomly distributed in time with respect to the transmitted high-voltage pulses. During the data post-processing, the E-FISH signal waveforms (with timing determined from the respective photodiode signals monitoring the laser pulses) are synchronized with respect to the discharge pulse by placing them into "time bins" $0.2 \mathrm{~ns}$ long, accumulating about 20 to 250 shots per time bin. Shots within each time bin are then integrated over time and averaged, as was done in our previous work [20,26]. This approach greatly accelerates the data acquisition and yields the sub-ns time-resolution of the data, in spite of the significant timing jitter (see Fig. 2). The stability of the laser output pulse energy, which is critical for the accuracy of the electric field inference from the second harmonic signal, is monitored by the photodiode. Based on the photodiode data (see Fig. 2), the laser pulse energy variation does not exceed a few percent. Care was taken to ensure that the PMT signal was not saturated. This was verified by confirming that the PMT voltage, as well as the temporal profile and the peak value of the signal, were within their acceptable limits, determined based on previous experiments.

To verify that the second harmonic signal scales quadratically with the axial electric field, the setup is modified for calibration such that an axial Laplacian field is sustained between two circular, parallel plate electrodes placed in the discharge tube $4 \mathrm{~mm}$ apart, with the laser beam passing between the electrodes. The electric field calibration data have been taken for all four operating pressures, 20, 40, 70, and $100 \mathrm{mbar}$. The results of the electric field calibration indicate that over this pressure range, most of the second harmonic signal induced by the electric field in the discharge originates within the quartz wall of the discharge tube, as discussed in greater detail in Section 3. For this reason, two alternative calibration techniques have been tested and found to produce consistent results. Firstly, for all pressures, the absolute value of the electric field was obtained by comparing the second harmonic signal with the current shunt signals, after the ionization wave has reached the grounded electrode, i.e. at the conditions when the axial electric field distribution along the discharge gap is known to be uniform [25]. In addition, the E-FISH signals obtained at 20 mbar were compared to the axial electric field profile calculated from the distribution of the electric potential along the discharge gap, obtained from capacitive probe measurements. From the calibration, the detection limit of the present E-FISH measurements is approximately $0.25 \mathrm{kV} / \mathrm{cm}$.

\section{Results and Discussion}

Figure 3(a) shows a 200 shot averaged broadband plasma emission image measured in a ns pulse discharge in nitrogen at $P=20$ mbar, $3.2 \mathrm{~ns}$ after the beginning of the voltage rise. The camera gate is $1.17 \mathrm{~ns}$, and the field of view is approximately $2.5 \mathrm{~cm}$ x $6.8 \mathrm{~cm}$. In Fig. 3(a), the high-voltage electrode is on the left, the grounded electrode is on the right, and the flow is left to right. It can be seen that the emission intensity in nitrogen is significantly higher near the discharge tube walls, which is illustrated further by plotting the radial distributions of the emission intensity 
in Fig. 3(b), at the axial locations indicated by a dashed line in Fig. 3(a). This result, which is consistent with our previous experimental and kinetic modeling studies of fast ionization wave discharges in nitrogen [16,27], shows that the ionization wave is propagating predominantly near the walls of the discharge tube. Kinetic modeling predictions [16] reveal that this occurs due to the higher electric field near the wall, caused by the jump in permittivity, and due to the secondary photo-electron emission from the wall irradiated by the UV photons from the plasma. At higher pressures, when the electron transport becomes slower, this effect is more pronounced. It is interesting to note that such radial non-uniformity is also present in a capillary discharge sustained in a significantly smaller diameter quartz tube of $1.5 \mathrm{~mm}$ [27].

Figure 4 plots the second harmonic signal calibration lines obtained by measuring the Laplacian field maintained between two circular, parallel plate electrodes $D=20 \mathrm{~mm}$ in diameter and $d=4 \mathrm{~mm}$ apart placed in the discharge tube, at four different pressures. The voltage pulse is applied to the electrodes by the same ns pulse generator as was used to sustain the discharge in the tube. The calibration data are taken only during the voltage rise, before breakdown occurs in the tube. The voltage rise is determined from the incident and reflected pulses measured by the current shunt connected to the high voltage electrode, while the occurrence of breakdown is inferred from the rise of the transmitted voltage pulse, measured by the current shunt connected to the grounded electrode. For all four pressures, the square root of the second harmonic signal is proportional to the applied electric field (see Fig. 4), as expected. For a plane wave approximation and for a uniform electric field, the line-of-sight integrated intensity of the horizontally polarized secondharmonic signal, $I_{x}^{(2 \omega)}$, is [28],

$$
I_{x}^{(2 \omega)} \sim\left[\chi_{x x}^{(3)} E_{x} I_{x}^{(\omega)}\right]^{2}\left[\frac{\sin (\Delta k \cdot L / 2)}{\Delta k / 2}\right]^{2} .
$$

In Eq. (1), $E_{x}$ is the horizontal (axial) component of the electric field, $I_{x}^{(\omega)}$ is the intensity of the horizontally polarized laser beam, $\chi_{x x}^{(3)}$ is the third-order nonlinear susceptibility (in gases, proportional to the number density), $L$ is the length of the region where the electric field is applied, in the direction of the laser beam, and $\Delta k$ is the difference between the fundamental and the second harmonic wave vectors. At the present conditions, the coherence length in nitrogen at $P=20-100$ mbar, $L_{c, N 2}=\pi /\left(2 k_{\omega}-k_{2 \omega}\right) \approx 60-300 \mathrm{~cm}$, and in ambient air, $L_{c, \text { air }} \approx 6 \mathrm{~cm}$, is very long, such that the phase matching is not expected to affect the signal in the plasma or in ambient air, where it is therefore proportional to $L^{2}$. On the other hand, the coherence length in quartz (fused silica) is much shorter, $L_{c, F S} \approx 10 \mu \mathrm{m}$, such that the wall thickness strongly affects the electric field induced second harmonic signal generated in the wall.

From Eq. (1), the square root of the second harmonic signal in a gas is proportional to the number density. However, from the data plotted in Fig. 4, it is readily apparent that varying the pressure in the tube by a factor of 5 has essentially no effect on the signal. This indicates that most of the signal is generated not in the nitrogen within the tube, but either in the quartz wall or in the atmospheric pressure air outside the tube, induced by the electric field extending beyond the space between the electrodes. The third order susceptibility of fused silica at $1064 \mathrm{~nm}$ is nearly 4 orders of magnitude larger compared to that of atmospheric pressure air, $\chi_{F S}^{(3)}=2 \cdot 10^{-22} \mathrm{~m}^{2} / \mathrm{V}^{2}[29,30] \mathrm{vs}$. $\chi_{\text {air }}^{(3)}=3 \cdot 10^{-26} \mathrm{~m}^{2} / \mathrm{V}^{2}[30,31]$. The second harmonic signal from the ambient air would be comparable to that originating within the discharge tube wall only if it is produced over a distance 
of $L \sim L_{c, F S} \cdot \chi_{F S}^{(3)} / \chi_{\text {air }}^{(3)} \sim 7 \mathrm{~cm}$. This is extremely unlikely, since the laser beam diameter over this distance is increasing from $\approx 0.5 \mathrm{~mm}$ at the wall to $\approx 4 \mathrm{~mm}$, and since the electric field outside of the discharge tube is decreasing. We therefore conclude that at the present conditions, the signal is likely to be generated in the wall.

This estimate is consistent with the results of Ref. [18], which show that for tight focusing configurations (i.e. for the lens focal distance of $15 \mathrm{~cm}$ and $30 \mathrm{~cm}$ ), the second harmonic signal is also generated from the regions beyond the confocal beam parameter, such that the contribution of the electric-field induced signal from the tube wall could be dominant at the present conditions. Basically, the Laplacian field calibration indicates that a relatively slow reduction of the signal intensity with the distance from the beam focal point is offset by the much higher nonlinear susceptibility of fused silica. This suggests that in the present experiments the second harmonic signal is sensitive to the electric field generated within the wall of the discharge tube, such that the plane-to-plane Laplacian field calibration may well be inaccurate.

An alternative calibration method employed in the present work is based on a significant body of previous electric field measurements using calibrated back current shunts [24] and capacitive probes $[25,15]$. The results of these measurements show that after the ionization wave has reached the grounded electrode and the discharge gap is closed, the axial electric field distribution along the discharge tube becomes uniform and close to the difference between the potentials on the electrodes divided by the discharge gap. The potential difference between the electrodes, after the ionization wave "touchdown", is inferred from the two current shunt signals, one measuring the potential waveform in the incident and reflected voltage pulses (before the highvoltage electrode), and the other measuring the potential in the transmitted pulse (after the grounded electrode). For the absolute calibration, the square root of the second harmonic signal intensity is matched to the electric field obtained from the current shunt waveforms during the pulse voltage reduction, well after the wave reaches the grounded electrode. The confidence in the absolute calibration of the present measurements may well be increased by comparison with kinetic modeling, predicting electric field distributions both in the plasma and in the wall of the discharge tube, as has been done previously for electric field measurements using an electro-optic probe [32].

Figure 5 plots a typical data set (square root of the second harmonic signal before the calibration is applied), taken in the ionization wave discharge in nitrogen at $P=100$ mbar. In this figure, black symbols indicate the raw single-shot data points taken during a 2-hour long run, and red symbols show the averaged data points in 200 ps time bins, obtained during the data postprocessing. The error bars indicate statistical uncertainty (standard deviation using the Student's tdistribution) of the data. From Fig. 5, different phases of the discharge development, labeled in the figure, are readily identified and well understood, based on the previous lower-fidelity electric field measurements by capacitive probes [15,23-25], theoretical analysis, and kinetic modeling predictions [16]. The initial gradual rise of the signal corresponds to the Laplacian field generated ahead of the approaching ionization wave after it is initiated near the high-voltage electrode during the pulse-voltage rise. The peak occurs when the ionization front arrives at the measurement location, and the electric field begins to decrease rapidly, due to the charge separation in the plasma generated in the wave. The electric field behind the wave is reduced considerably, but remains above the detection limit at all pressures tested, such that the electrons created in the wave front are transported toward the high-voltage electrode, generating the conduction current. The secondary rise of the electric field occurs after the wave front arrives at the grounded electrode 
(cathode), the secondary emission is generated from the cathode, and the discharge transitions into a quasi-steady-state regime. Finally, the electric field decays to near detection limit as the applied voltage is reduced to zero.

Figure 6(a-d) plots time-resolved, absolute axial electric field distributions in a fast ionization wave discharge in nitrogen at $P=20-100$ mbar, shown together with the current shunt signal used for absolute calibration. It can be seen that the electric field waveform obtained by the E-FISH measurements matches the current shunt waveform after the moment when the wave reaches the grounded electrode (see Fig. 6(a-c), although at $P=20$ mbar the signal-to-noise ratio in the quasi-steady-state discharge becomes significantly lower (see Fig. 6(d)). At these low-pressure conditions, the emission from the quasi-steady-state discharge and the afterglow becomes much stronger, such that subtracting it from the PMT signal improves the signal-to-noise. The effect of the plasma emission in the afterglow is also visible but less pronounced at $P=40$ mbar (see Fig. 6(c)), where it has not been subtracted from the signal. At $P=20 \mathrm{mbar}$, additional comparison has been made with the electric field measured by a calibrated capacitive probe, the technique described in detail in our previous work [15,25] (see Fig. 6(d)). Note that the capacitive probe measurements match very well the current shunt waveform in the quasi-steady-state discharge. However, it is readily apparent that the electric field peak in the ionization wave front, measured by the capacitive probe, is not fully resolved compared to the E-FISH data (the apparent peak is wider and the maximum value is lower). This behavior is expected, since the effective time resolution of the capacitive probe ( 1.5-3.0 ns), limited by its spatial sensitivity, is up to an order of magnitude lower compared to that of the present E-FISH diagnostic (200 ps).

Comparison of the data taken at different pressures exhibits several dominant trends. First, in the entire pressure range, the peak electric field in the ionization wave exceeds the quasi-steadystate value established after the wave reaches the grounded electrode, by up to a factor of 4 to 5 . This is much higher compared to a relatively modest electric field overshoot, $\sim 10 \%$, detected in a ns pulse discharge generated in 100 Torr of nitrogen across a relatively short gap of $13 \mathrm{~mm}$, where the wave does not fully develop [19]. Second, the peak value of the axial electric field in the wave front exhibits a fairly weak pressure dependence, $E_{\text {peak }}=8-11 \mathrm{kV} / \mathrm{cm}$ (compare Figs. 6(a-d)). The highest reduced electric field, $E / N \approx 2000 \mathrm{Td}$, is produced at the lowest pressure of $P=20 \mathrm{mbar}$, as expected (see Fig. 7). The gas density used in computing the reduced field assumes room temperature, which is anticipated given the low deposited energy density of this discharge. Third, reducing the pressure from 100 to 20 mbar, while keeping the discharge pulse voltage waveform the same, steepens the ionization wave front considerably, as illustrated in Fig. 7. At 100 mbar, the full width at half maximum of the transient electric field peak in the wave front is $3.0 \pm 0.2 \mathrm{~ns}$, while at 20 mbar it is reduced to $1.0 \pm 0.2 \mathrm{~ns}$, and remains fully resolved by the present diagnostic. This behavior is most likely due to the faster electron impact ionization at a significantly higher reduced electric field in the wave, as well as the more rapid electron transport behind the wave front, resulting in plasma self-shielding on a sub-ns time scale. Finally, the wave speed increases considerably as the pressure is reduced, from $V=0.4 \mathrm{~cm} / \mathrm{ns}$ at $100 \mathrm{mbar}$ to $V=1.6 \mathrm{~cm} / \mathrm{ns}$ at 20 mbar. The wave speed at the present conditions was determined from the time-resolved plasma emission intensity distribution measurements, such as that shown in Fig. 3, with a relative uncertainty of $2 \%$. The difference between the wave speed measured on the tube centerline and near the wall did not exceed 5\%. The wave speed values are indicated in the legend of Fig. 7.

\section{Summary}


In this work, non-intrusive, time-accurate, absolute measurements of the electric field in a fast ionization wave discharge sustained over a wide range of pressures are obtained for the first time. Since the present results are based on the detection of the electric field within the discharge tube wall, they demonstrate that ps second harmonic generation using the present approach may be employed for electric field measurements at the conditions when the detection of the second harmonic signal generated directly in the plasma is challenging, i.e. (a) in low-pressure discharges, (b) in discharges sustained in small diameter capillary tubes, and (c) in discharges sustained in gases with very low nonlinear polarizability, such as helium. These results also show that the contribution of the discharge cell walls into the net electric-field induced second harmonic signal has to be identified and accounted for, e.g. using the electrostatic calibration over a wide range of pressures. Since the signal generated within the wall during the discharge is induced by the electric field, it cannot be easily subtracted from the net second harmonic signal, unlike the zero-field background.

In principle, the temporal resolution of the present measurements is limited only by the laser pulse duration, $30 \mathrm{ps}$, and may be improved further by using an oscilloscope with a higher sampling rate. In low and moderate pressure FIW discharges, this opens a possibility of detection and analysis of non-local electron kinetics effects in the presence of rapidly varying, high peak value reduced electric fields. Finally, with additional calibration, the same approach can be used to measure the radial component of the electric field in the ionization wave front. The present results can also be used for assessing the predictive capability of kinetic models of fast ionization waves generating breakdown on ns and sub-ns time scale.

\section{Acknowledgments}

The work was partially supported by French National Research Agency, ANR (ASPEN Project), LabEx Plas@Par and the French-Russian international laboratory LIA KaPPA 'Kinetics and Physics of Pulsed Plasmas and their Afterglow'. The experiments were conducted at the Centre Laser de l'Université Paris Sud (CLUPS/LUMAT FR 2764). The authors are thankful to Ali Mahjoub, Bruno Dufour and Pascal Pariset for technical assistance, and to Dr. Michel Broquier and Dr. Gilles Grégoire for helping setting up these experiments. The support of Prof. Adamovich by the Ecole Polytechnique Gaspard Monge Visiting Professor (GMVP) Program is gratefully acknowledged.

\section{References}

1. A.Yu. Starikovskiy and N.L. Aleksandrov, "Plasma-assisted ignition and combustion", Progress in Energy and Combustion Science 39 (2013) 61

2. S.M. Starikovskaia, "Plasma-assisted ignition and combustion: nanosecond discharges and development of kinetic mechanisms", Journal of Physics D: Applied Physics 47 (2014) 353001

3. Y. Ju and W. Sun, "Plasma assisted combustion: Dynamics and chemistry", Progress in Energy and Combustion Science 48 (2015) 21

4. G. Petitpas, J.-D. Rollier, A. Darmon, J. Gonzalez-Aguilar, R. Metkemeijer, and L. Fulcheri, "A Comparative Study of Non-Thermal Plasma Assisted Reforming Technologies", 
International Journal of Hydrogen Energy 32 (2007) 2848

5. L.M. Martini, N. Gatti, G. Dilecce, M. Scotoni, and P. Tosi, "Laser induced fluorescence in nanosecond repetitively pulsed discharges for $\mathrm{CO}_{2}$ conversion", Plasma Physics and Controlled Fusion 60 (2018) 014016

6. S.B. Leonov, I.V. Adamovich and V.R. Soloviev, "Dynamics of near-surface electric discharges and mechanisms of their interaction with the airflow", Plasma Sources Science and Technology 25 (2016) 063001

7. D. Graves, "Low temperature plasma biomedicine: A tutorial review", Physics of Plasmas, 21 (2014) 080901

8. S.M. Starikovskaia, A.Yu. Starikovskii and D.V. Zatsepin, "The development of a spatially uniform fast ionization wave in a large discharge volume", Journal of Physics D: Applied Physics 31 (1998) 1118

9. B.-D. Huang, E. Carbone, K. Takashima, X.-M. Zhu, U. Czarnetzki, and Y.-K. Pu, "The effect of the pulse repetition rate on the fast ionization wave discharge", Journal of Physics D: Applied Physics, in press, https://doi.org/10.1088/1361-6463/aabf2d

10. S. Pancheshnyi, D.A. Lacoste, A. Bourdon and C.O. Laux, "Ignition of propane-air mixtures by a repetitively pulsed nanosecond discharge", IEEE Transactions on Plasma Science 34 (2006) 2478

11. S.A. Shcherbanev, N.A. Popov, S.M. Starikovskaia, "Ignition of high pressure lean $\mathrm{H}_{2}$ :air mixtures along the multiple channels of nanosecond surface discharge", Combustion and Flame 176 (2017) 272

12. L.M. Vasilyak, S.V. Kostyuchenko, N.N. Kudryavtsev, I.V. Filyugin, "Fast ionisation waves under electrical breakdown conditions", Physics-Uspekhi 37 (1994) 247

13. S.M. Starikovskaia, N.B. Anikin, S.V. Pancheshnyi, D.V. Zatsepin and A.Yu. Starikovskii, "Pulsed breakdown at high overvoltage: development, propagation and energy branching", Plasma Sources Science and Technology 10 (2001) 344

14. A.V. Klochko, J. Lemainque, J.P. Booth and S.M. Starikovskaia, "TALIF measurements of oxygen atom density in the afterglow of a capillary nanosecond discharge", Plasma Sources Science and Technology 24 (2015) 025010

15. N.B. Anikin, S.M. Starikovskaia, and A.Yu.Starikovskii, "Polarity effect of applied pulse voltage on the development of uniform nanosecond gas breakdown", Journal of Physics D: Applied Physics 35 (2002) 2785

16. K. Takashima, I.V. Adamovich, Z. Xiong, M.J. Kushner, S. Starikovskaia, U. Czarnetzki, and D. Luggenhölscher, "Experimental and Modeling Analysis of Fast Ionization Wave Discharge Propagation in a Rectangular Geometry", Physics of Plasmas 18 (2011) 083505

17. T. Darny, J.-M. Pouvesle, V. Puech, C. Douat, S. Dozias, and E. Robert, "Analysis of conductive target influence in plasma jet experiments through helium metastable and electric field measurements", Plasma Sources Science and Technology 26 (2017) 045008

18. A. Dogariu, B.M. Goldberg, S. O'Byrne, and R.B. Miles, "Species-independent femtosecond localized electric field measurement", Physical Review Applied 7 (2017) 024024 
19. B.M. Goldberg, T.L. Chng, A. Dogariu, and R.B. Miles, "Electric field measurements in a near atmospheric pressure nanosecond pulse discharge with picosecond electric field induced second harmonic generation", Applied Physics Letters 112 (2018) 064102

20. M. Simeni Simeni, Y. Tang, K. Frederickson, and I.V. Adamovich, "Electric Field Distribution in a Surface Plasma Flow Actuator Powered by Ns Discharge Pulse Trains", Plasma Sources Science and Technology 27 (2018) 104001

21. M. Simeni Simeni, Y. Tang, Y.-C. Hung, Z. Eckert, K. Frederickson, and I.V. Adamovich, "Electric Field in Ns Pulse and AC Electric Discharges in a Hydrogen Diffusion Flame", Combustion and Flame 197 (2018) 254

22. B. Goldberg, S. Reuter, and A. Dogariu, "1-D Electric Field Measurements Using Field Induced Second Harmonic Generation", $60^{\text {th }}$ Annual Meeting of the APS Division of Plasma Physics, November 5-9, 2018, Portland, OR, USA

23. N.B. Anikin, S.V. Pancheshnyi, S.M. Starikovskaia, and A.Yu.Starikovskii, "Breakdown development at high overvoltage: electric field, electronic level excitation and electron density", Journal of Physics D: Applied Physics 31 (1998) 826

24. N.B. Anikin, S.M. Starikovskaia, and A.Yu.Starikovskii, "Study of the oxidation of alkanes in their mixtures with oxygen and air under the action of a pulsed volume nanosecond discharge", Plasma Physics Reports 30 (2004) 1028

25. A.V. Klochko, S.M. Starikovskaia, Zh. Xiong, and M.J. Kushner, "Investigation of capillary nanosecond discharges in air at moderate pressure: comparison of experiments and 2D numerical modelling”, Journal of Physics D: Applied Physics 47 (2014) 365202

26. B.M. Goldberg, I. Shkurenkov, S. O’Byrne, I.V. Adamovich, and W.R. Lempert, "Electric Field Measurements in a Dielectric Barrier Nanosecond Pulse Discharge with SubNanosecond Time Resolution”, Plasma Sources Science and Technology 24 (2015) 035010

27. N.D. Lepikhin, N.A. Popov and S.M. Starikovskaia, "Fast gas heating and radial distribution of active species in nanosecond capillary discharge in pure nitrogen and $\mathrm{N}_{2}: \mathrm{O}_{2}$ mixtures", Plasma Sources Science and Technology 27 (2018) 055005

28. I. J. Bigio, R. S. Finn, and J. F. Ward, "Electric-field induced harmonic generation as a probe of the focal region of a laser beam", Applied Optics 14 (1975) 336

29. H. Guillet de Chatellus and E. Freysz, "Measurement of the third-order susceptibility of glasses by EFISH of femtosecond pulses", Optics Express 9 (2001) 586

30. U. Gubler and C. Bosshard, "Optical third-harmonic generation of fused silica in gas

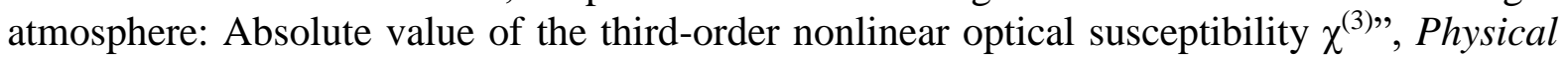
Review E 61 (2000) 10702

31. D.P. Shelton and J.E. Rice, "Measurements and Calculations of the Hyperpolarizabilities of Atoms and Small Molecules in the Gas Phase", Chemical Reviews 94 (1994) 3

32. A. Bourdon, T. Darny, F. Pechereau, J.-M. Pouvesle, P. Viegas, S. Iséni, and E. Robert, "Numerical and experimental study of the dynamics of a $\mu$ s helium plasma gun discharge with various amounts of $\mathrm{N}_{2}$ admixture", Plasma Sources Science and Technology 25 (2016) 035002 


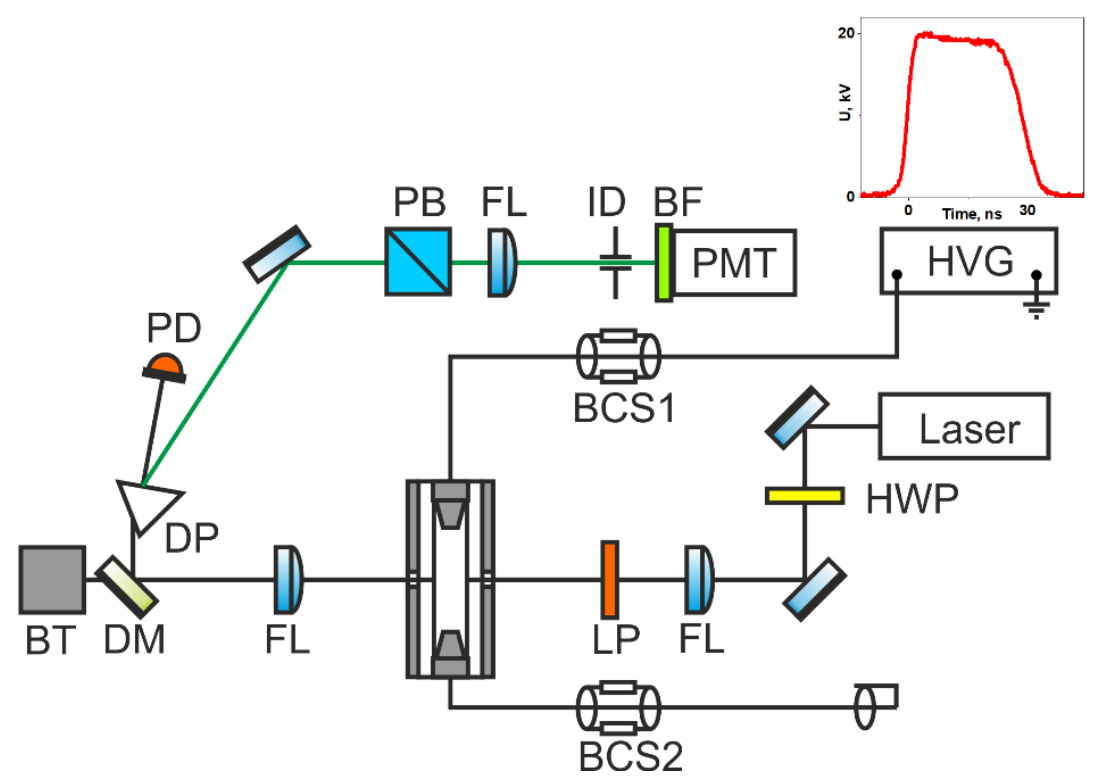

Figure 1. Schematic of the experimental apparatus and ps second harmonic generation diagnostic. BT: beam trap; DM: dichroic mirror (532 nm reflecting, $1064 \mathrm{~nm}$ transmitting); DP: dispersive prism; PD: photodiode; FL: plano-convex lens; PB: $532 \mathrm{~nm}$ polarizer; ID: iris diaphragm; BF: $532 \mathrm{~nm}$ bandpass filter; PMT: photomultiplier tube; LP: long pass filter; HWP: $1064 \mathrm{~nm}$ half-wave plate; HVG: high voltage generator; BCS1, BCS2: back current shunts before the high-voltage electrode and after the grounded electrode, respectively. The inset shows the voltage waveform on the highvoltage electrode, top view of the discharge tube is shown.

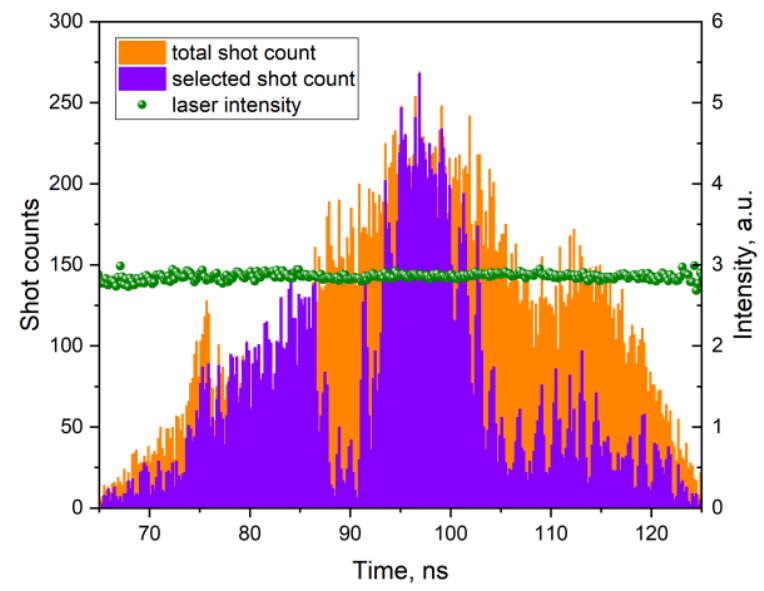

Figure 2. Histograms showing distributions of 40,000 laser shots (orange) and data points where the EM noise was sufficiently low (purple), plotted together with the laser pulse energy distribution over $0.2 \mathrm{~ns}$ time bins (green). 


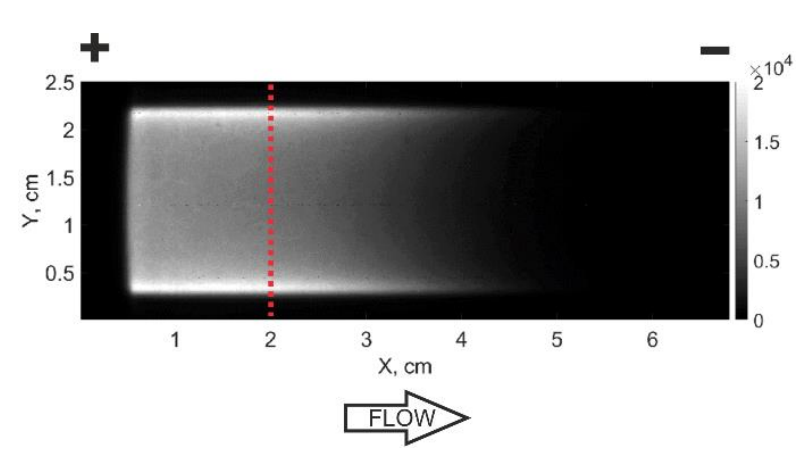

(a)

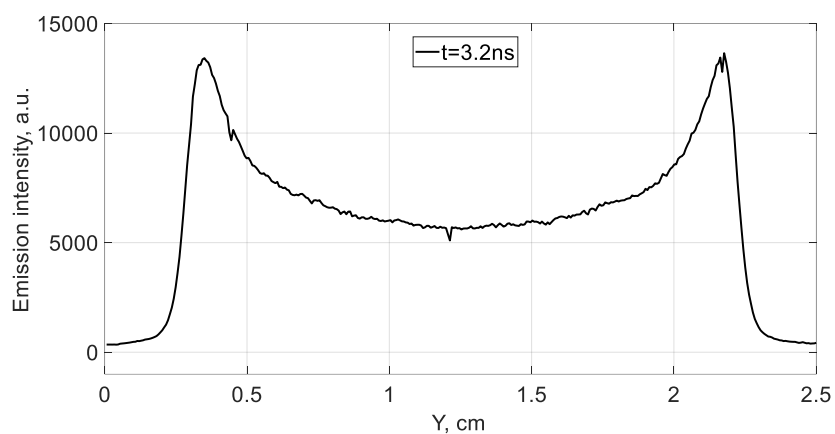

(b)

Figure 3. (a) ICCD camera image of a broadband emission from the plasma. The electrode polarity, the flow direction, and the axial location where the emission intensity distribution across the discharge tube is plotted are shown. (b) Plasma emission intensity distribution at the indicated axial location. Nitrogen, pressure $20 \mathrm{mbar}$, flow rate $10 \mathrm{sccm}$, discharge tube inside diameter $20 \mathrm{~mm}$, camera gate $1.17 \mathrm{~ns}$. 


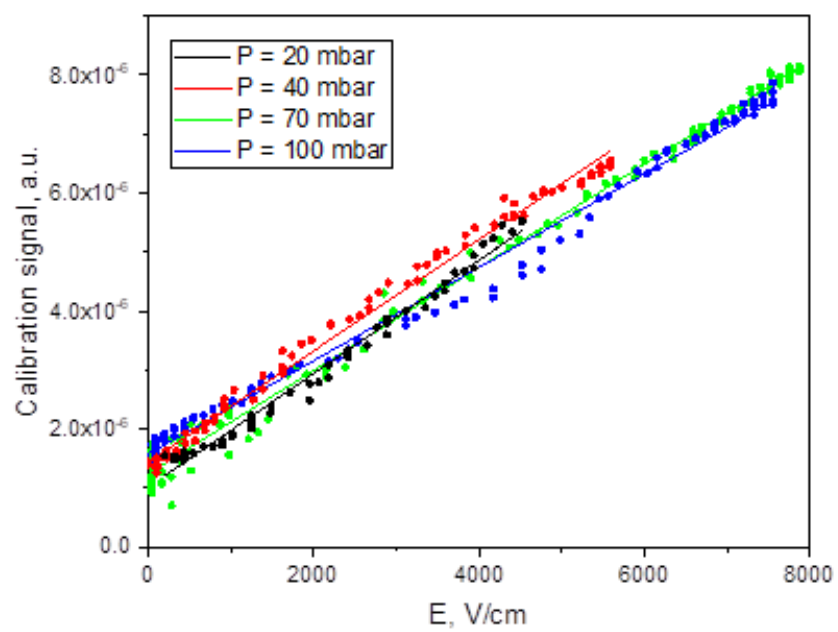

Figure 4. Second harmonic signal calibration lines obtained by measuring the Laplacian field maintained between two parallel plate electrodes $20 \mathrm{~mm}$ in diameter and $4 \mathrm{~mm}$ apart placed in the discharge tube, at $\mathrm{P}=20-100$ mbar.

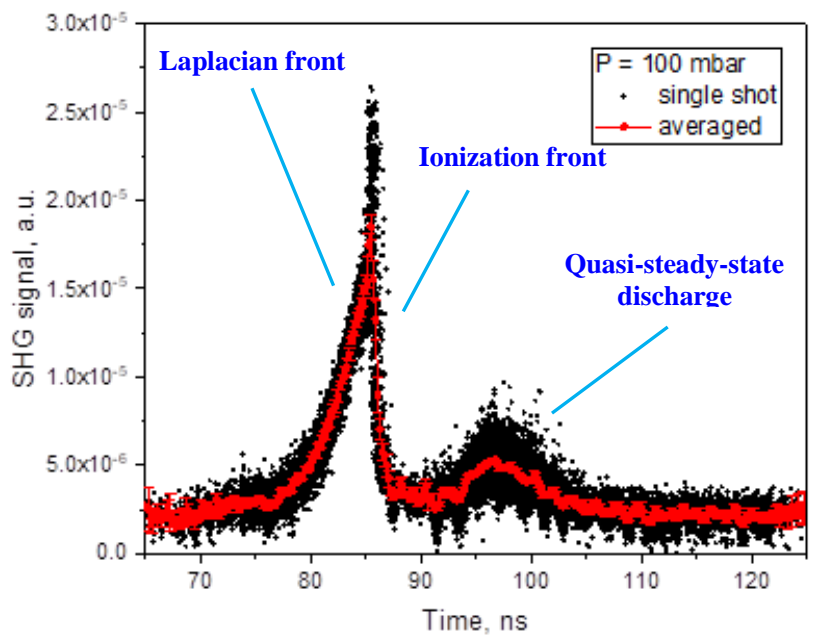

Figure 5. Typical second harmonic signal data set, taken in a ns pulse discharge in nitrogen at $\mathrm{P}=100$ mbar, before the calibration is applied. Black symbols indicate single-shot data points, red symbols are averaged data points in 200 ps time bins. Error bars indicate statistical uncertainty (standard deviation) of the data. 

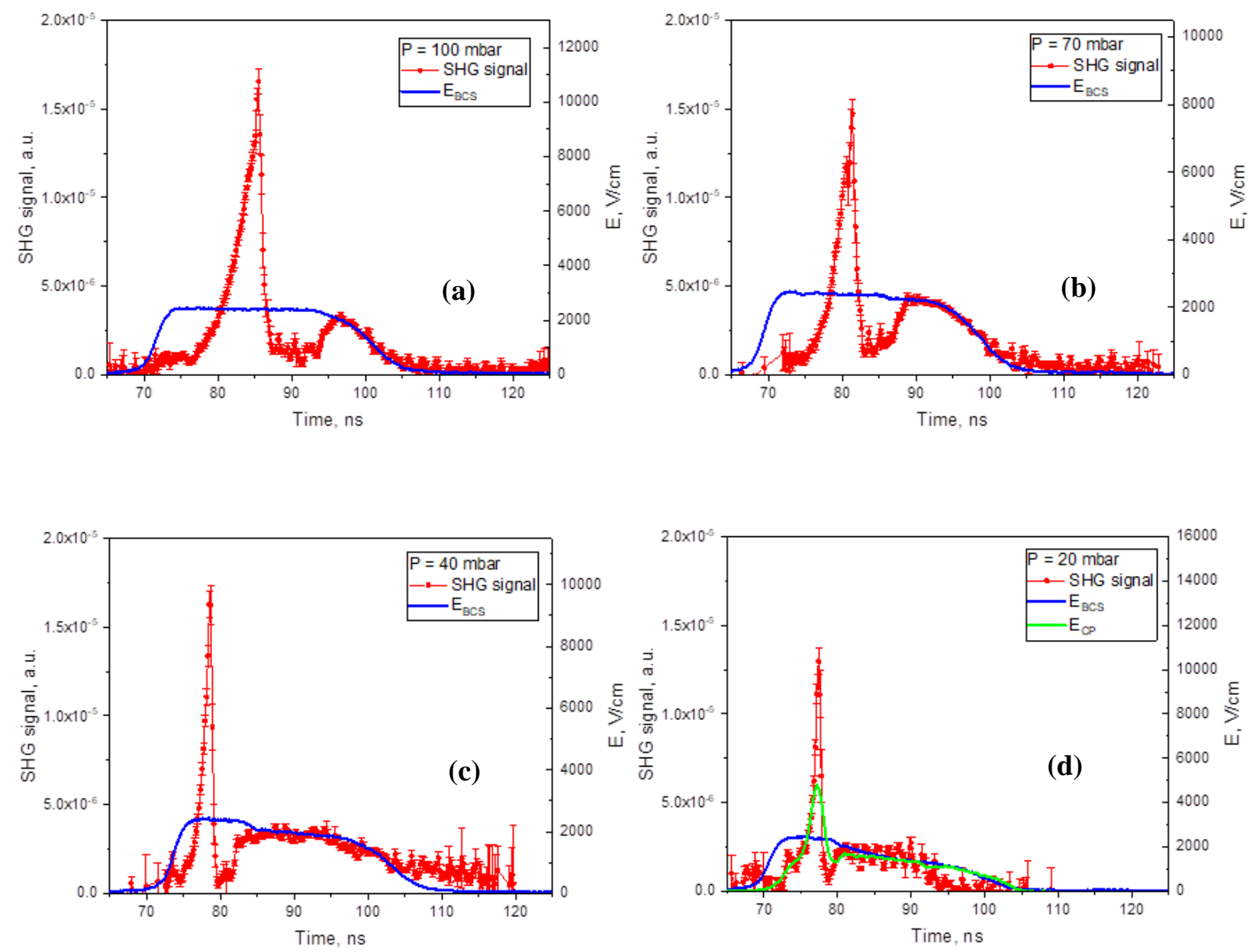

Figure 6. Electric field in a fast ionization wave discharge in nitrogen at $\mathrm{P}=20-100$ mbar, plotted together with the current shunt signal used for absolute calibration $\left(\mathrm{E}_{\mathrm{BCS}}\right)$. Electric field inferred from the capacitive probe measurements $\left(\mathrm{E}_{\mathrm{CP}}\right)$ is also plotted at $\mathrm{P}=20$ mbar. 


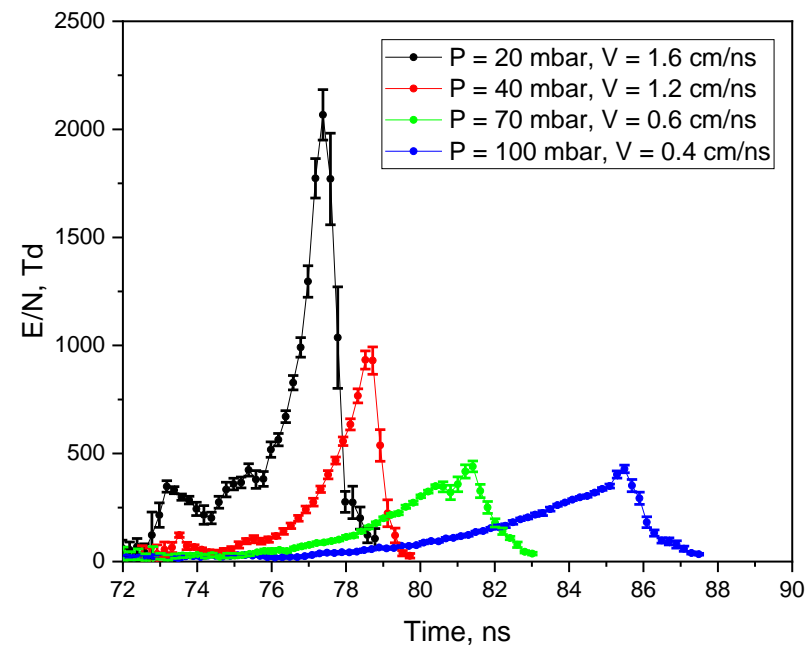

Figure 7. Reduced electric field measured in the front of a fast ionization wave discharge in nitrogen at $\mathrm{P}=20-100$ mbar, plotted on the same scale. 